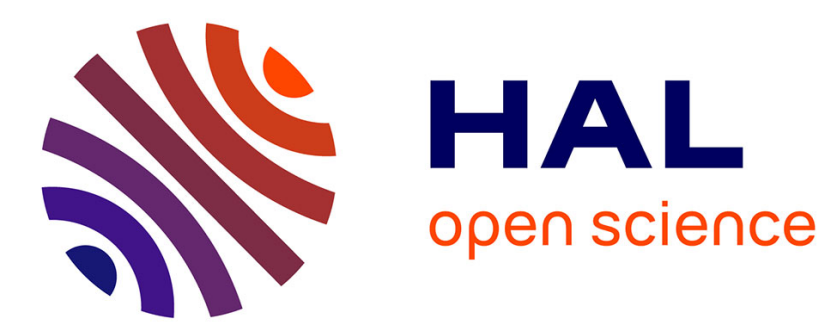

\title{
Postures, manipulations, déambulations : comprendre la grammaire anglaise autrement
}

Jean-Rémi Lapaire, Pascale Etcheto

\section{To cite this version:}

Jean-Rémi Lapaire, Pascale Etcheto. Postures, manipulations, déambulations : comprendre la grammaire anglaise autrement. La nouvelle revue de l'adaptation et de la scolarisation, 2010, 49, pp.45-58. halshs-01629056

\section{HAL Id: halshs-01629056 \\ https://shs.hal.science/halshs-01629056}

Submitted on 6 Nov 2017

HAL is a multi-disciplinary open access archive for the deposit and dissemination of scientific research documents, whether they are published or not. The documents may come from teaching and research institutions in France or abroad, or from public or private research centers.
L'archive ouverte pluridisciplinaire HAL, est destinée au dépôt et à la diffusion de documents scientifiques de niveau recherche, publiés ou non, émanant des établissements d'enseignement et de recherche français ou étrangers, des laboratoires publics ou privés. 


\title{
Postures, manipulations, déambulations : comprendre la grammaire anglaise autrement
}

\author{
Jean-Rémi LAPAIRE ${ }^{1}$ \\ et Pascale ETCHETO 2 \\ Université Michel de Montaigne-Bordeaux 3
}

Résumé : Le fonctionnement de la langue est dans une large mesure postural : nous prenons position, nous adoptons des points de vue, des distances et des attitudes vis-à-vis des êtres, des idées et des phénomènes. Dans cet article, nous montrons qu'il est possible d'explorer physiquement les postures sociales et mentales qu'exprime la grammaire anglaise, au moyen d'une gestualité métaphorique exécutée en cours de langue ou en atelier " grammaire et expression corporelle".

Mots-clés : Cognition - Espaces de conceptualisation - Gestes métaphoriques - Grammaire anglaise Incorporation du sens - Symbolisme gestuel.

New attitudes to linguistic description: using metaphoric motion and manipulation to make sense of English grammar

Summary: Much about language seems to be ruled by posture: speakers take stands, have viewpoints, approach issues from different angles, stand close or keep their distance. This paper shows how mental and physical stances may be studied, using various forms of gesture symbolism. The body-motion conceptualisation of grammar may be performed in traditional classroom settings or during special langue and movement workshops.

Key words: Cognition - Conceptual space - Embodied meaning - English grammar - Gesture symbolism Gestural metaphors.

$\mathbf{P}$ RENDRE la parole, c'est nécessairement adopter une posture physique, mentale et sociale face à des personnes, à des choses, à des expériences ou à des idées. Au travers du langage, nous nous rapprochons ou prenons des distances, nous adoptons des angles et des perspectives. Quel rôle joue la grammaire dans l'élaboration et le codage de ces postures? Des notions aussi fondamentales que le temps, l'aspect, la modalité (assertion, emphase, négation, interrogation, hypothèse) renvoient toutes à un positionnement du locuteur ou à

1. Jean-Remi.Lapaire@u-bordeaux3.fr

2.Pascale.Etcheto@u-bordeaux3.fr 
une attitude vis-à-vis de ce qu'il énonce. Cela se traduit, à l'oral, par des postures corporelles indéfiniment variables mais identifiables:

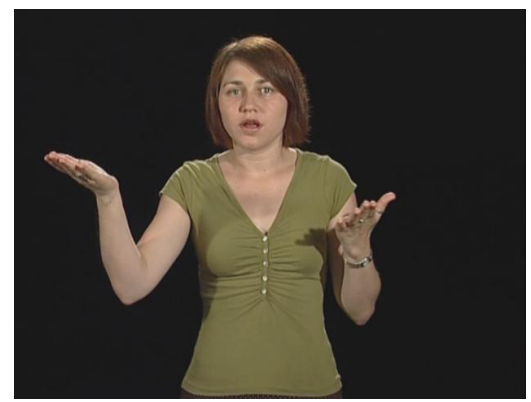

Figure 1 - Posture interrogative (Lapaire \& Masse, 2006)

"Qui a fait ça? Où, quand, comment, pourquoi?" Gestualité co-verbale spontanée.

L'objectif de cet article est de monter qu'une sensibilisation à la dimension posturale de la grammaire peut être entreprise en atelier, à l'occasion de l'étude d'une langue étrangère. Divers moyens sont envisageables. Le plus évident consiste à s'appuyer sur des enregistrements authentiques pour mettre en rapport certaines constructions avec l'attitude physique des sujets, en établissant des liens entre posture corporelle et posture socio-cognitive. Mais l'observation est fastidieuse et la systématisation hasardeuse car la gestualité co-verbale est variable, polysémique et multidimensionnelle (McNeill, 1995; Kendon, 1997; Harrison, 2009).

Une deuxième solution, que nous ébauchons ici, consiste à explorer et à jouer les postures que nous exprimons au travers de la langue: engagement, manipulation, blocage, balancement, équilibrage, contournement... II ne s'agit pas de reproduire les vrais attitudes et les vrais gestes accompagnant la vraie parole (bien qu'on puisse s'en inspirer) mais de mobiliser le corps pour mieux comprendre le fonctionnement social et l'architecture conceptuelle de la langue vivante étrangère (en l'occurrence I'anglais), tout en effectuant un travail syntaxique et phonologique rigoureux:

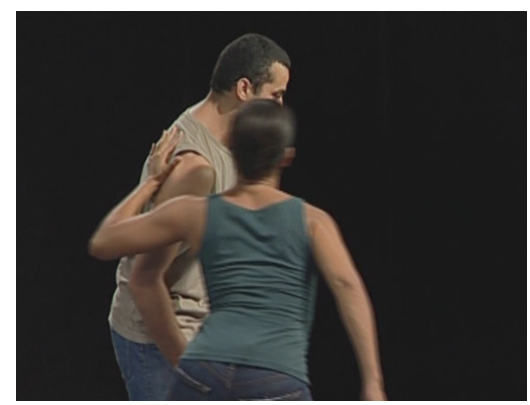

Figure 2 - Posture de contrôle associée à must (Lapaire \& Masse, 2006) "Mettre la pression ", "pousser à l'action ", "vaincre une résistance " Gestualité explicative inventée.

Une approche visuelle kinesthésique de ce type permet d'inscrire la réflexion métalinguistique dans le corps et dans l'espace. Cette incorporation et cette 
spatialisation de mécanismes abstraits a en outre l'avantage de mettre en lumière ce qu'est la grammaire:

- un lieu de rencontre et de découverte de l'autre (Aden \& Anderson, 2005), avec tout ce que cela implique de placement, de mise en relation, d'interaction;

- un lieu de conceptualisation et de symbolisation de l'expérience, qui force à adopter un point de vue sur les événements, à décomposer et à linéariser les situations évoquées, à identifier des actants et à leur attribuer des rôles.

Après avoir caractérisé notre approche, nous suggérons quelques modes de mise en $œ u v r e$. Les exemples visuels sont empruntés au DVD Grammar in Motion (Lapaire \& Masse, 2006) et à l'atelier croisé Grammaire et expression corporelle en cours d'expérimentation à l'université de Bordeaux 3.

\section{POSTURES, MANIPULATIONS ET DÉAMBULATIONS}

Si on se met à l'écoute de la langue ordinaire, on ne peut manquer d'être frappé par la régularité avec laquelle le corps et l'activité corporelle sont invoqués:

- pour parler de la vie sociale (ex. pousser, manipuler, forcer (la main), (se) traîner, (se) bouger, entrer en contact, toucher);

- pour parler de la vie mentale (ex. "saisir (une idée), voir (ce qu'on veut dire), avancer (dans une recherche), suivre (une explication), esquiver ou contourner (une difficulté), déplacer un problème »).

Dans la plupart des cas, cette imagerie corporelle opère à un niveau strictement symbolique car le corps physique ne s'engage pas réellement. Quelqu'un qui manipule I'opinion ou qui se repose sur ses acquis, ne manipule rien et ne s'allonge nulle part. Pourtant, la langue parle bien d'un corps, que nous appelons corps imaginaire lou symbolique) de la cognition (Lapaire, 2008). Non seulement la langue évoque ce corps invisible, mais elle l'anime pour lui faire jouer un répertoire codé de scènes: mains (imaginaires) qui saisissent le sens des choses, yeux (imaginaires) qui voient ce quelqu'un veut dire, jambes (imaginaires) qui reculent face à l'obstacle, etc. Ainsi la langue a-t-elle élaboré une physiologie imaginaire de la cognition et de l'interaction sociale dont les organes dominants sont les mains, les yeux et les jambes. Ces organes opèrent sur des objets invisibles, placés dans des espaces de conceptualisation. Dans peser le pour et le contre (weighing options), ce n'est pas le corps physique mais le corps symbolique qui tient dans le creux des mains des choses (les idées, les argumentations) et qui les met en balance.

Il arrive pourtant que le corps physique de l'énonciateur reproduise, sur un mode à la fois iconique et métaphorique (McNeill, 1992), I'activité fictive du corps symbolique. Cela arrive durant la parole et se manifeste au travers de la gestualité co-verbale spontanée. En prononçant peser le pour et le contre il n'est pas rare de voir l'énonciateur reproduire un geste simplifié de pesée, chaque main symbolisant un plateau de la balance, sur lesquels semblent posés d'invisibles objets. L'existence de cette gestualité, simultanément abstraite et concrète, prouve qu'il est possible de représenter l'activité du corps symbolique, en joignant le geste iconique à la parole métaphorique. Dans l'exemple ci-dessous, le corps symbolique de la cognition est représenté: après avoir construit une hypothèse, on voit qu'elle ne tient pas debout et on l'écarte définitivement: 


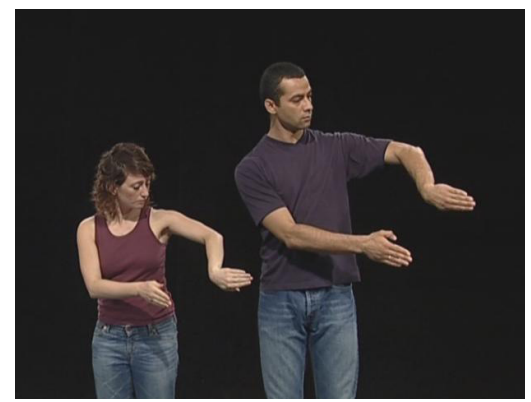

Figure 3 - Posture de mise à l'écart codée par can't (Lapaire \& Masse, 2006) "Balayer, écarter une hypothèse (qui ne tient pas debout)"

Ex. She can't be 60! She can't have been pleased with the result!

La posture cognitive adoptée par le corps symbolique de la cognition est codée par le corps physique des danseurs. L'image reproduite ici est une capture d'écran du DVD Grammar in Motion (Lapaire \& Masse, 2006). Une cinquantaine de notions et de constructions grammaticales y sont expliquées et illustrées au moyen de métaphores gestuelles de ce type, exécutées par des danseurs professionnels. Ces gestes physiques, appelés KineGrams, donnent corps aux mouvements imaginaires que convoquent les LogoGrams: petites formules imagées, traduisant en langage physique les mécanismes et les significations linguistiques (ex. écarter une hypothèse):

Mécanisme grammatical Traduction en langage physique Représentation gestuelle Marqueur + valeur LogoGram KineGram

L'espace qui entoure le corps dans ces scènes est un espace de conceptualisation: il peut représenter le domaine du temps, de l'expérience, de la réalité, de la connaissance ou encore l'espace social. Bien que plus large et extensible que l'espace gestuel ordinaire de la communication, il possède la même plasticité symbolique: on peut tout lui faire signifier et absolument tout y représenter. De même, les objets (invisibles) qui sont désignés ou manipulés, représentent un nombre illimité de choses, concrètes ou abstraites, animées ou inanimées, définies ou indéfinies.

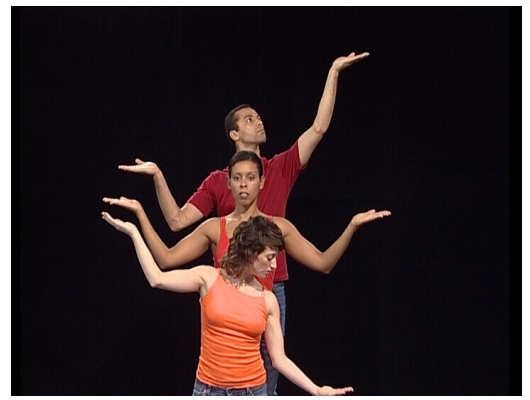

Figure 4 - Posture cognitive de la comparaison (Lapaire \& Masse, 2006) "Placer (deux choses) au même niveau " (as... as...);

"Placer au-dessus " (more /-er than...); " placer au-dessous " (not as... as...) 
La facilité avec laquelle il est possible de créer des "concepts visuels " (Arnheim, 1969) à partir de notions les plus abstraites confirme qu'il existe bien " des équivalences sensorielles de toutes les notions théoriques ". La banque de ressources visuelles que nous avons créée fournit une amorce qui peut être utilisée de multiples façons. On peut reproduire, simplifier, enrichir ou concevoir d'autres pratiques corporelles signifiantes pour analyser la grammaire des langues. Le dispositif décrit ci-après constitue un exemple possible.

\section{VERS DE NOUVELLES PRATIQUES MÉTALINGUISTIQUES?}

L'atelier grammaire et expression corporelle monté à l'université de Bordeaux 3 associe un professeur de linguistique anglaise (Jean-Rémi Lapaire) et une professeure de danse (Pascale Etcheto). Chaque atelier (1 h30) est travaillé en amont par les deux intervenants: caractérisation du phénomène étudié, recension des formes linguistiques, identification des postures sociales et cognitives, essai de traduction en langage corporel (comme équilibre, déséquilibre, extensions pour le comparatif et superlatif; se déplier, se replier, aller vers l'autre et revenir sur soi pour les pronoms réfléchis ou réciproques). Au terme de la réflexion, des propositions sont élaborées: affichage des objectifs de la séance et plan de déroulement des séquences. Comme dans toute pratique corporelle, un soin particulier est apporté aux phases d'entrée et de sortie du dispositif: les sons et les mouvements pratiqués lors de l'échauffement sont liés aux thèmes de la séance. Ainsi, des déplacements dans le gymnase préparent la mise en place des notions de chemin ou de trajectoire de l'action (lines of action, action paths), de finalité ou de but (assimilés à des destinations). Le croisement des corps et des regards prépare utilement les notions de rencontre et de contact. Des vocalisations élémentaires peuvent également être pratiquées pour préparer un travail articulatoire et intonatif ultérieur sur les marqueurs (-self/-selves, -s, -ed, -ing, 't , etc.), utile et apprécié. Nous présentons, dans les pages qui suivent, trois cas concrets d'étude portant successivement sur la négation, l'aspect et le temps.

\section{La négation}

II existe trois grands procédés pour exprimer la négation en anglais: choix de mots dont le sens est intrinsèquement négatif (hate, refuse, deny, oppose), adjonction d'un suffixe négatif à une base lexicale (un-welcome, mis-understood, de-regulated, dis-interested, dys-functional, point-less), emploi d'une construction grammaticale spécifique de type auxiliaire + not (/ don't understand) ou no + N (/ have no clue ${ }^{3}$ ). Ces éléments formels peuvent être travaillés en amont ou en aval de l'exploration gestuelle des scripts ou modèles cognitifs de la négation (décrits plus loin). Durant l'échauffement vocal, un travail ludique peut être entrepris autour de:

- I'articulation et la couleur des suffixes un-, mis-, de-, dis-/dys- et -less,

- I'accentuation et I'humeur des mots négatifs (selon qu'on les charge ou non d'affects),

- l'intonation des constructions syntaxiques négatives.

3. Givón (2001), à qui nous empruntons cette classification, emploie respectivement les termes : instrinsic negation, morphological negation, syntactic negation. 
Mais on peut également attendre la fin des activités d'expression corporelle pour vocaliser, en s'appuyant sur les notions et les sensations mises en évidence durant la phase d'exploration sensorielle et gestuelle des trois modèles cognitifs qui structurent notre compréhension de la négation (Lapaire, 2006). Ces modèles sont: - le rejet ${ }^{4}$ d'une chose qui est proposée (placée devant soi/devant les yeux). On décline une offre (No thanks. I'm afraid I can't), on écarte ou délaisse une idée. Inversement, l'acceptation ${ }^{5}$ consiste à prendre, à se saisir de la chose et à la faire sienne.

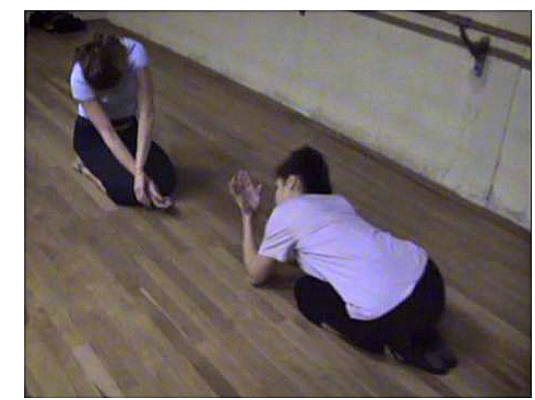

Figure 5 - Atelier croisé " grammaire et expression corporelle " "Offrir, proposer; accepter, rejeter, délaisser".

- I'obstacle entravant le chemin qui mène à l'action. On s'oppose, on résiste (/ won't go for this) et ce faisant on tente de bloquer.

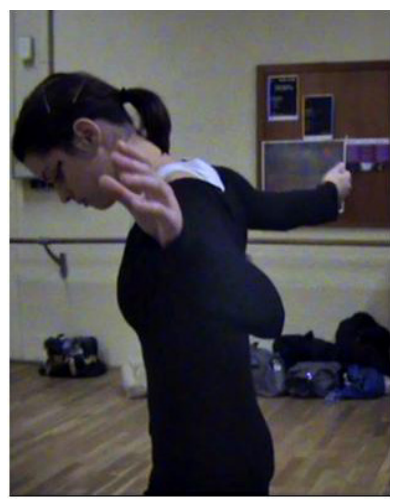

Figure 4 - Atelier croisé "grammaire et expression corporelle " "Faire obstacle, se détourner, s'interposer" (angl. standing in the way).

- la destruction de ce qui est faux, inacceptable (This isn't true. You're wrong. It's not $X$ but $Y$.

Un lecture scénique de la négation révèle que la négation n'est pas une simple affaire de formes et de structures: le véritable enjeu est la construction de scènes avec

4. Du latin reicere, dérivé jacere "lancer, jeter".

5. Du latin acceptare, dérivé de capere "prendre ". 
des actants. Qui rejette, qui bloque, qui élimine? Avec quelle énergie? Lorsqu'il y a interposition, comment barre-t-on la route de l'autre? Avec brutalité ou ménagement? Lorsqu'on subit la négation, quelles options a-t-on? Insister? Forcer? Négocier? Se détourner? Contourner? Reculer? Disparaître?

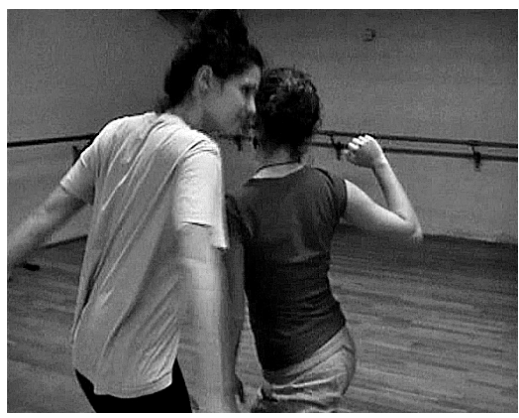

Figure 5 - Atelier croisé " grammaire et expression corporelle " "Contourner l'obstacle" (angl. getting around an obstacle).

Le travail sur les acteurs de la négation débouche nécessairement sur un questionnement de fond sur le fonctionnement social de la négation. Pour ne pas blesser ou offenser par un refus trop direct, des structures existent, comme I'm afraid (she's out). Maybe you should (call back later). Des équivalents physiques de cette posture de refus courtois ont pu être trouvés et les participants ont reconnu qu'ils avaient pris conscience physiquement du phénomène.

\section{L'aspect}

À cette catégorie sont traditionnellement rattachées des formes lexicales ou grammaticales reflétant un point de vue sur le déroulement de l'action, selon qu'on voit celle-ci de l'intérieur ou de l'extérieur, qu'on la considère comme bornée ou non bornée, achevée ou inachevée, saisissable en bloc ou articulée en phases (initiale, médiane, finale). Ainsi, lorsque nous lisons le panneau Sold by Foxtons Estate, nous interprétons le participe sold comme une forme aspectuelle du verbe sell, qui indique que toutes les étapes du processus de vente (selling one's home) ont été franchies avec succès jusqu'à la signature finale de l'acte ${ }^{6}$. L'aspect est dit accompli (ou perfectif) car le processus a été mené à son terme. Implicites, les phases initiales et intermédiaires restent à l'arrière-plan car ce que sold donne à voir ou met en relief est l'état achevé (ou résultant) du processus de vente: vendue (sold), la maison n'est plus à vendre (for sale) ou en vente (au prix de...) (selling [for...]). $C^{\prime}$ est à dessein que nous employons un vocabulaire à la fois spatial et visuel pour parler de l'aspect car la notion de point de vue est métaphoriquement inscrite dans le mot, qui est issu du latin aspectus (regard, apparence, perspective). Dans cet arrangement visuel de la conceptualisation (Langacker, 2000), le sujet cognitif est métaphoriquement assimilé à un observateur (angl. viewer, observer) et l'action à une chose inscrite dans l'espace, qui s'offre au regard. Comme le note Janda (2004),

6. LogoGram proposé (Lapaire, 2006): " Du début à la fin, on a fait tout le chemin ". 
l'action-chose peut être conçue comme un objet discret (simple ou composite) ou comme une substance (compacte ou extensible). Rares sont pourtant les présentations qui exploitent ce substrat visuel et matériel de l'aspect, comme si le retour à des primitives sensorielles constituait une menace de régression conceptuelle. Comme si relier une notion linguistique au corps, à l'espace et à la matière bloquait toute élévation intellectuelle, alors que c'est exactement le contraire qui s'impose. C'est en sollicitant l'expérience et l'intelligence sensori-motrices du monde, c'est en exploitant les formes de symbolisme verbal ou gestuel qui leur sont associées, qu'on arrive à faire voir ou à faire saisir les mécanismes abstraits de la langue. $C^{\prime}$ 'est en stimulant les sens qu'on permet à la grammaire de faire sens et c'est en stimulant l'imagerie mentale qu'on permet à un véritable imaginaire grammatical de se développer (Lapaire, 2002). Ainsi peut-on convoquer l'image d'un petit objet dur qui rebondit contre une surface, tels une balle ou un marteau de porte, pour keep asking, keep telling, keep complaining (aspect itératif/répétitif). Ou encore l'image d'une pâte souple et homogène qu'on étire pour rendre compte de go on working, go on and on (aspect continu/duratif/progressif). Ailleurs encore, c'est la rotation d'une main ou d'un doigt traçant une spirale qui sera sollicitée pour représenter la notion de continuité, comme cela s'observe dans la gestualité co-verbale spontanée où le progressif et l'itératif sont susceptibles de recevoir ce type de codage gestuel (Harrison, 2009):

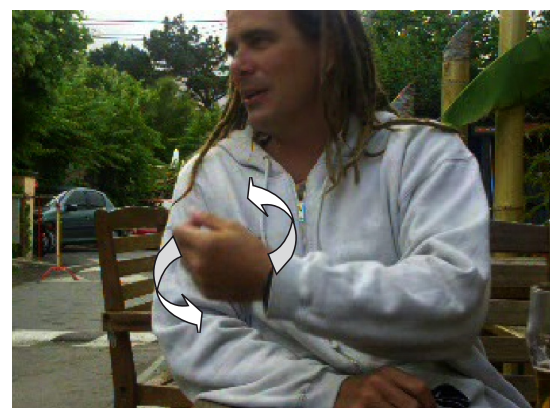

Figure 6 - "Geste cyclique " spontané accompagnant certaines expressions du progressif en anglais (Harrison, 2009: 223).

Dans les ateliers croisés que nous proposons, il est envisageable de manipuler fictivement des objets et des substances invisibles, de tracer des trajectoires avec les mains pour expliquer un certain nombre de notions aspectuelles, en s'inspirant éventuellement des KineGrams présentés dans Grammar in Motion (Lapaire \& Masse, 2006). Cependant, il nous semble plus intéressant d'engager le corps tout entier, en pratiquant une gestualité plus libre et des déplacements plus amples dans l'espace. En nous appuyant sur l'idée que toute action implique un changement d'état, métaphoriquement assimilé à un changement de lieu (Lakoff \& Johnson, 1999), nous demandons aux participants de fixer un point à atteindre dans la salle. Entre l'endroit où ils se trouvent (point de départ de l'action) et l'endroit où il se rendent (terme de l'action), ils doivent exécuter une séquence gestuelle libre dont la seule contrainte est de former un tout (l'entier de l'action). Les différentes phases 
du développement (états intermédiaires) doivent s'enchaîner et tendre vers un aboutissement. L'exercice est répété plusieurs fois, dans le silence ou sur un fond sonore, avec des consignes différentes:

- Porter son attention sur la phase initiale: préparation, amorce du premier geste (sensibilisation à l'aspect inchoatif/ingressif présent dans les constructions start + -ing, begin + to);

- S'attacher à la fluidité de ses gestes, se sentir pris dans un déroulement, dans un flot ininterrompu, développer, laisser émerger puis maintenir (travail sur l'aspect continu/progressif associé aux emplois prototypiques de be + -ing, continue + -ing; go on + -ing);

- S'affranchir de la conscience du début ou de la fin, se sentir totalement dans l'action, exister au travers d'elle (travail sur l'immersion dans un processus codé par be + -ing et mise en évidence de la notion d'effectuation pure marquée par -ing, "le simple fait de faire ", walking, laughing, singing...).

Des formes verbales correspondant aux notions travaillées peuvent être proposées en soutien. On les fait alors défiler mentalement en exécutant les séquences ou on les répète lentement à voix basse, en dilatant l'articulation gestuelle et sonore: moving... dreaming... breathing...

L'atelier permet aussi de travailler diverses postures, statiques ou dynamiques, isolées ou enchaînées, pour prendre conscience des différences et des complémentarités existant entre états et processus. Plus généralement, le montage des séquences autorise une spatialisation de notions grammaticales fondamentales comme l'agentivité, l'intention, la volition, le moyen, le résultat: Vers où ai-je envie d'aller?, Quels moyens est-ce que je me donne pour y parvenir? Qu'est-ce qu'arriver au bout d'une chose? Quelle posture finale (état résultant) est-ce que je me trouve? Suis-je tonique ou alangui? Fier ou effondré ? Tendu ou apaisé? Seul ou entouré ? Cette dernière série de questions accompagne le débat sur les notions d'accomplissement et d'état résultant, couramment associées au participe passé ( $V$-en, done, made, gone, walked, found, written...), au parfait (have $+-e n)$ et au passif (be $+-e n)$ :

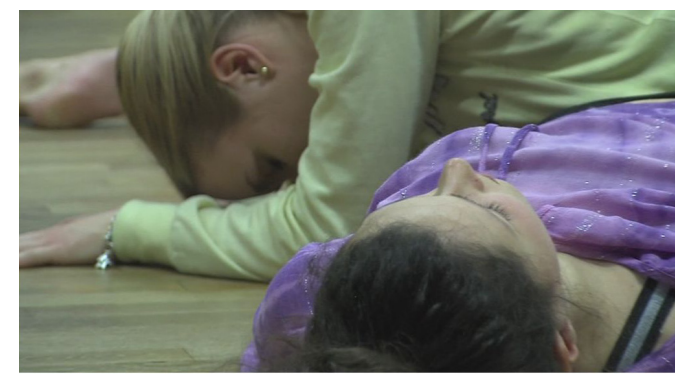

Figure 7 - Atelier croisé " grammaire et expression corporelle " (Lapaire \& Etcheto, 2010) "Arriver au bout de l'action ": exploration des postures et sensations associées à l'état final. 


\section{Temps: de l'ancrage déictique à l'intersubjectivité}

La langue institue un rapport postural au temps qu'il est aisé de mettre en évidence dans une pratique corporelle de la réflexion métalinguistique. Nous nous tenons debout dans l'ici et le maintenant du présent:

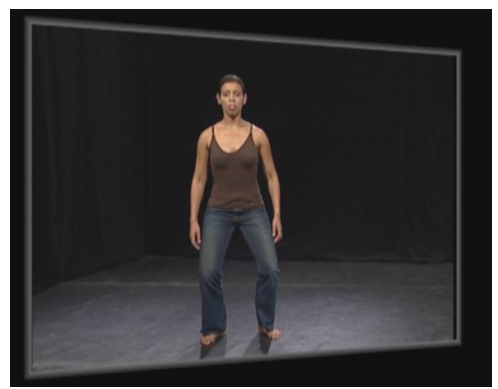

Figure 8 - Posture de l'ancrage déictique (Lapaire \& Masse, 2006)

"Sous mes pieds (here), le présent (now)".

À partir de cet ancrage déictique fondamental, nous sommes capables de nous retourner sur notre passé, de remonter mentalement le cours du temps (yesterday, a month ago, back in 1910) et plus généralement d'explorer (visuellement, physiquement) le temps. Le souvenir est assimilé à une vision à rebours (rétrospection) - on revoit les choses (métaphoriquement situées derrière nous) - ou à un retour : on remonte, on voyage dans le passé. Une organisation comparable existe pour le futur: on scrute I'avenir, assimilé à un horizon, on prévoit ce qui arrivera (prospection). On se dirige aussi vers le futur ou alors on laisse l'avenir venir de lui-même (le jour viendra où..., [the] time will come when...).

Qu'il s'agisse de passé ou d'avenir, on note que les yeux et les jambes sont les organes les plus sollicités. Pour associer les deux modes de conceptualisation visuel et moteur - tout en impliquant davantage d'organes, on peut pratiquer des torsions, des inclinaisons de buste, des fentes avant, associées à des regards et à des extensions de bras, tout en prenant soin de garder un ou deux pieds dans le présent, afin de signifier que l'ancrage premier reste signifiant même si on s'en éloigne : on peut alors se retourner sur son passé, on se pencher sur son avenir. Ce travail postural sur notre attitude face au temps, précède utilement une réflexion plus ciblée sur les formes lexicales et grammaticales impliquées (prétérit, go-future,

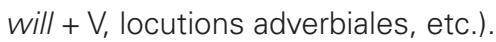

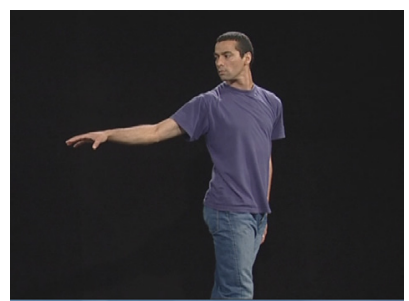

Figure 9 - Posture rétrospective

Travail sur le passé, la prétérition, ago (Lapaire \& Masse, 2006). 

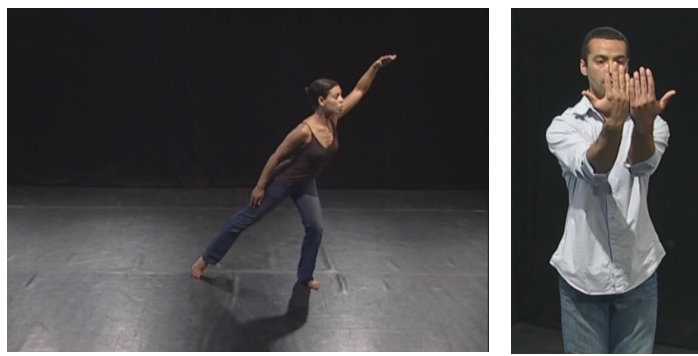

Figure 10 - Postures prospectives (Lapaire \& Masse, 2006) Travail sur le futur: scruter, se pencher, faire surgir une vision (will).

Comme on le voit, la réflexion porte à la fois sur la cognition langagière générale et sur des formes linguistiques particulières. A l'instar de ce qu'on observe dans la langue des signes, les gestes et les postures possèdent un grand pouvoir de compression. Dès lors, il devient possible de créer des raccourcis visuels saisissants, qui se substituent à de longues explications analytiques. Pourquoi la particule to dans have to, need to, ought to, want to, going to? Parce que ce qui est visé, désiré, envisagé, etc. se trouve là-bas devant soi au bout d'une ligne invisible:

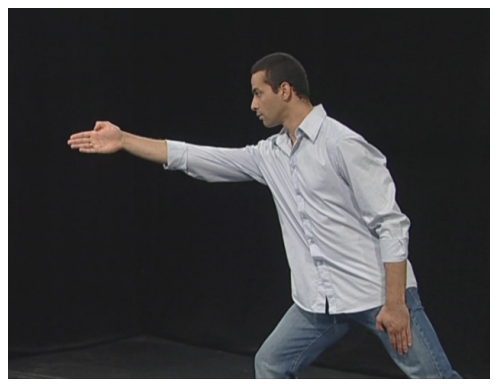

Figure 11 - To: "viser l'action! " (Lapaire \& Masse, 2006).

L'étude de la temporalité ne se laisse pas réduire aux seuls repérages dans le temps. Les ateliers sont un lieu privilégié de mise en évidence d'autres dimensions pertinentes, notamment dans le domaine de la prétérition. Quitter mentalement la réalité présente, avec le passé (went, called, lived), n'est pas forcément synonyme de remonter dans le temps. On utilise aussi certaines formes du passé pour rêver, pour construire et explorer des mondes virtuels ou encore pour atténuer la force d'un propos ou adoucir une demande. Les grammaires anglaises classiques parlent alors d'imaginative use of tense et de tentative use of the past: Suppose we went there... If I lived there... ; I wanted to ask you something... I thought I might be a good idea to... I wanted to ask you about... Pour expliquer ce phénomène, nous nous asseyons à même le sol, face aux participants (disposés en demi-cercle). Nous avons recours à des gestes des bras et de la main: nous plaçons dans l'oblique, légèrement au-dessus du niveau de référence de la réalité, les espaces du rêve ou de l'hypothèse. Nous suggérons manuellement que le prétérit allège, distancie, arrondit ce qu'un impératif ou un présent aurait (im)posé brutalement: 


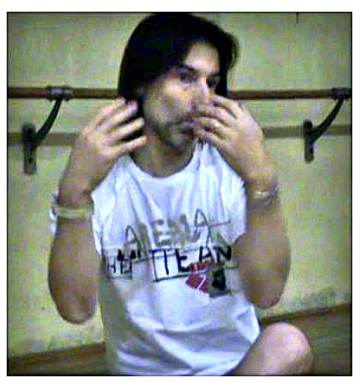

Figure 12 - Atelier croisé " grammaire et expression corporelle " (Lapaire \& Etcheto, 2010) Explication de la " douceur " et de la "rondeur " apportées par le prétérit.

Dans les exercices posturaux, nous travaillons essentiellement à mettre en évidence la dimension interactionnelle du temps: parler au passé non pour évoquer un moment du passé mais pour mieux agir socialement dans le présent. L'exercice le plus convaincant, est celui qui oppose stratégie coercitive à stratégie persuasive. Les participants travaillent par paires. L'un d'eux doit conduire l'autre à l'action (symbolisée par un point de la salle à atteindre). Dans un premier temps, il a le droit de toucher, de prendre la main, de guider de façon proche et directe. Dans un second temps, il n'a pas le droit de toucher, garde davantage de distance, tourne autour, enrobe tandis que l'autre, initialement perplexe ou résistant, se laisse entraîner à l'action. Au terme de cet exercice, des constructions correspondant aux deux stratégies sont présentées et travaillées (avec une attention particulière prêtée à l'intonation).

\section{CONCLUSION}

Solliciter les sens et la motricité pour mieux comprendre le fonctionnement de la langue ne va pas de soi : ni les pratiques institutionnelles, ni la configuration matérielle des salles de cours ne s'y prêtent. Quiconque entend incorporer et spatialiser la réflexion métalinguistique doit donc être prêt à bousculer les inerties, à braver les résistances, à dominer ses propres craintes, à trouver des partenaires (éducation physique, arts de la scène) et à investir d'autres lieux:

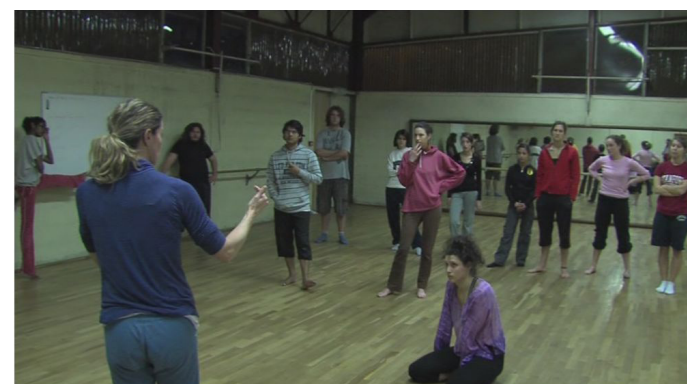

Figure 13 - Atelier croisé " grammaire et expression corporelle " (Lapaire \& Etcheto, 2010), "Ceci est (bien) un cours de grammaire!"

Aucun obstacle n'est cependant insurmontable : les personnes et les lieux existent, l'institution est capable de soutenir l'innovation, les inhibitions intérieures sautent dès qu'on accepte l'idée d'un format hybride d'enseignement, verbal et non verbal. 
En associant la motricité et la réflexion, la sensation et l'intellection, l'instruction et l'expérimentation, les consignes strictes et l'improvisation, l'étude de notions générales et l'appropriation de formes particulières, on se met en situation de penser différemment le fonctionnement du langage. D'autres idées viennent, d'autres mots surgissent, d'autres façons de penser apparaissent et de nouvelles postures d'enseignement et d'apprentissage s'installent :

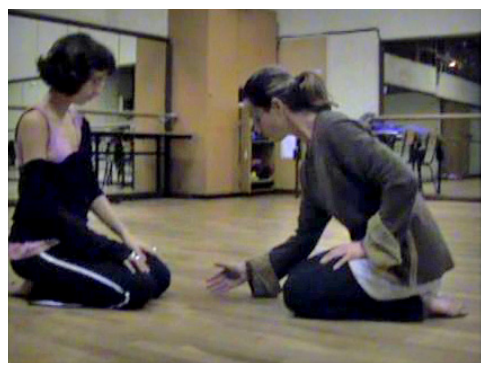

Figure 14 - Atelier croisé " grammaire et expression corporelle " (Lapaire \& Etcheto, 2010) Expliquer, comprendre et apprendre différemment. Ici la dialectique de l'offre et de l'acceptation.

Contraints de s'engager physiquement, les participants s'impliquent et participent à une co-construction des savoirs sur la langue. Même si tous n'accrochent pas, aucun ne décroche totalement. Chacun étant libre de s'agiter durant les exercices, il règne une rare qualité d'attention durant les discussions et les bilans.

La logique dans laquelle nous inscrivons cette recherche-action est celle de l'émergence. Chaque séance est l'occasion d'évolutions et d'ajustements. Le dispositif présenté dans ces lignes n'est donc pas stabilisé et n'est pas forcément destiné à être importé tel quel. II a en revanche vocation à encourager des modes de réflexion plus incorporés sur le fonctionnement des langues vivantes. II nous reste bien sûr à en évaluer les bénéfices, selon un protocole d'observation rigoureux. Au-delà des gains de compréhension escomptés, il sera intéressant de déterminer dans quelle mesure une forme de réflexion plus sensorielle et intégrative (bouger, ressentir, interagir, penser), créatrice de concepts visuels marquants, laisse une trace intime et durable. Pour l'heure, nous invitions les professeurs de langue à nous rejoindre dans l'invention d'autres espaces et d'autres pratiques métalinguistiques, en contrepoint des lieux et des modes d'instruction plus traditionnels, qu'il ne s'agit pas de délaisser.

\section{Références bibliographiques}

ADEN (Joëlle), JOEL (Anderson), Le drama pour une approche interculturelle des langues, Selected papers and presentations from the $24^{\text {th }}$ Annual Colloquium. Learning by Acting in a Professional Environment, 2005.

http://www.tesol-france.org/Colloquium05.php 
HARRISON (Simon), Grammar, Gesture, and Cognition. The Case of Negation in English / Grammaire, gestualité et cognition : le cas de la négation en anglais, Thèse bilingue pour le doctorat de l'université Michel de Montaigne-Bordeaux 3, 2009. GIVÓN (Talmy), Syntax, volume I, John Benjamins, Philadelphia /Amsterdam, 2001. JANDA (Laura), "A metaphor is search of a source domain : The categories of Slavic aspect," Cognitive Linguistics, 15-4, 471-527, 2004.

KENDON (Adam), "Gesture," Annual Review of Anthropology, Vol. 26, 109-128, 1997. LAKOFF (George), MARK (Johnson), Philosophy in the Flesh, Basic Books, New York, 1999.

LANGACKER (Ronald), Grammar and Conceptualization, Mouton de Gruyter, Berlin / New York, 2000.

LAPAIRE (Jean-Rémi), "Imaginative grammar", Cognitive Linguistics Today, 623-642. Edited by Barbara Lewandowska-Tomaszczyk and Kamila Turewicz, Peter Lang, Frankfurt, 2002, 19 pages.

LAPAIRE (Jean-Rémi), La grammaire anglaise en mouvement, Hachette, Paris, 2006. LAPAIRE (Jean-Rémi), "Negation, reification and manipulation in a cognitive grammar of substance", La Négation, Stéphanie Bonnefille et Sébastien Salbayre (Eds), Les Cahiers du GRAAT, n 35, 333-349. Tours, 2006.

LAPAIRE (Jean-Rémi), "Quand la pensée prend corps par la métaphore ", Dérives de la métaphore, Denis Jamet (ed.), 39-56, L'Harmattan, Paris, 2008.

LAPAIRE (Jean-Rémi), MASSE (Jean), Grammar in Motion, in Jean-Rémi Lapaire, La grammaire anglaise en mouvement, DVD encarté, Hachette, Paris, 2006.

MCNEILL (David), Hand and Mind. What Gestures Reveal About Thought, The University of Chicago Press, Chicago, 1992. 